\title{
Nestedness and Segmented Nestedness
}

\author{
Heikki Mannila \\ HIIT \\ Helsinki University of Technology and \\ University of Helsinki, Finland \\ mannila@cs.helsinki.fi
}

\author{
Evimaria Terzi \\ IBM Almaden Research Center \\ San Jose, CA, USA \\ eterzi@us.ibm.com
}

\begin{abstract}
Consider each row of a 0-1 dataset as the subset of the columns for which the row has an 1 . Then a dataset is nested, if for all pairs of rows one row is either a superset or subset of the other. The concept of nestedness has its origins in ecology, where approximate versions of it has been used to model the species distribution in different locations. We argue that nestedness and its extensions are interesting properties of datasets, and that they can be applied also to domains other than ecology.

We first define natural measures of nestedness and study their properties. We then define the concept of $k$-nestedness: a dataset is (almost) $k$-nested if the set of columns can be partitioned to $k$ parts so that each part is (almost) nested. We consider the algorithmic problems of computing how far a dataset is from being $k$-nested, and for finding a good partition of the columns into $k$ parts. The algorithms are based on spectral partitioning, and scale to moderately large datasets. We apply the methods to real data from ecology and from other applications, and demonstrate the usefulness of the concept.

Categories and Subject Descriptors: F.2.2 [ANALYSIS OF ALGORITHMS AND PROBLEM COMPLEXITY]: Nonnumerical Algorithms and Problems; H.2.8 [DATABASE MANAGEMENT]: Database ApplicationsData mining
\end{abstract}

General Terms: Algorithms, Experimentation, Theory

\section{Keywords}

nestedness, 0-1 matrices, presence/absence data

\section{INTRODUCTION}

The analysis of $0-1$ data is one of the recurring themes in data mining. One of the key issues in the area is to look for

\footnotetext{
*Work done while the author was at HIIT, University of Helsinki, Finland.
}

Permission to make digital or hard copies of all or part of this work for personal or classroom use is granted without fee provided that copies are not made or distributed for profit or commercial advantage and that copies bear this notice and the full citation on the first page. To copy otherwise, to republish, to post on servers or to redistribute to lists, requires prior specific permission and/or a fee.

KDD'07, August 12-15, 2007, San Jose, California, USA.

Copyright 2007 ACM 978-1-59593-609-7/07/0008 ...\$5.00. interesting concepts that would be useful to compute from such datasets.

In this paper, we consider the concept of nestedness of $0-1$ matrices. The concept has its origins in ecology; in the study of presence/absence matrices of sites (locations) and species. There, the nestedness hypothesis states that the species found in a site with few species should be a subset of the species found in a site with more species. That is, the sets of species found in the different sets should form a chain of subsets. Since the original publication [19], the nestedness concept has been studied a lot; see, e.g., [4, 11, 14] for some recent work. ${ }^{1}$

In general, consider each row of a 0-1 dataset as the subset of the columns for which the row has an 1 . Then a dataset is nested, if for all pairs of rows one row is either a superset or subset of the other. Equivalently, the dataset is nested if the rows and the columns can be reordered so that in the reordered matrix the $1 \mathrm{~s}$ in each row form a contiguous segment starting from the first column.

Nestedness is of course an extreme state, and there has also been some interesting work on quantifying the degree of nestedness in a dataset $[6,5,23,9,21]$. See Figure 1 for examples of datasets that are completely nested, almost nested, and very far from being nested. In the figure the rows and columns have been ordered so that the degree of nestedness is easy to see; in general, it is not easy to determine the degree of nestedness.
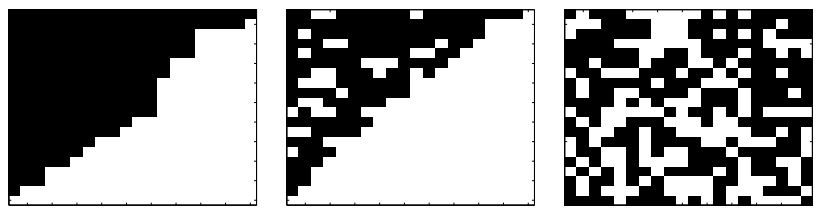

Figure 1: Examples of fully nested, almost nested and non-nested datasets. Black $=1$, white $=0$.

Nestedness is a concept that makes sense also for other types of datasets than ecological presence/absence data. Consider for example data about students and courses. Then, assuming that students follow a suggested study program with no electives, the set of courses that a student has taken is a sub- or a superset of the set of courses taken by another student. Deviations from this behavior tell us something about the students, the courses, or both.

\footnotetext{
${ }^{1}$ Note that this concept of nestedness is different from the concept of nestedness of model classes that is sometimes used in model selection.
} 
As another example, consider a set of documents about a single theme, say probability. Most documents contain the basic terms "probability", "random variable", "expectation" etc., while fewer contain terms such as "limit theorems", and still fewer documents contain terms such as "martingales". The set of terms in a document talking about martingales is most likely to be a superset of the set of terms in a document about the basic concepts of probability. In general, if the collection of documents is about a single topic and there are terms of different levels of difficulty or specialty, then the dataset can be expected to be nested.

Most datasets are not nested, but identifying the degree of their nestedness can give useful insight into the processes that produced the datasets.

In some cases the whole dataset might not be nested, but when limited to a subset of the variables, it is. Starting again with an ecological example, consider presence/absence data of species in sites distributed widely over space, say from north to south. Then the dataset as a whole is not nested: the northernmost and southernmost sites might each have many species, but very few species are found in both places. Thus, the subset/superset phenomenon does not occur.

However, the set of species can be partitioned into two sets such that when projected on those sets the data is nested. In this example the sets of species could be the ones that are prevalent in the south and those that are prevalent in the north. Such segmented nestedness has not, to our knowledge, been considered in the literature. See Figure 2 for an example.

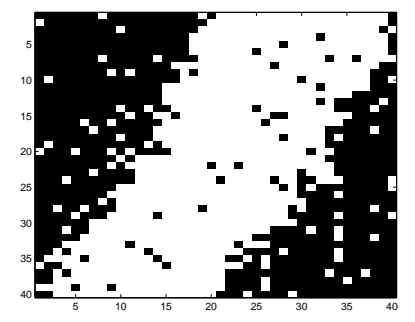

Figure 2: Example of a dataset that has segmented nestedness.

Segmented nestedness can be applied to other domains, as well. For example, in the course data we can find that the courses can be partitioned into two sets so that on those sets the data is nested; this would correspond to two different curricula. In the document example we could find that the terms can be divided into two sets in such a way that the use of the terms from both sets fall into a nested pattern.

In this paper we consider the concepts of nestedness and segmented nestedness, their use in data analysis, and the algorithmic properties of the concepts. We argue that nestedness and its extensions are interesting properties of datasets that can be applied also to domains other than ecology. We study the algorithmic properties of computing how far from being nested a dataset is. We then define the concept of $k$-nestedness: a dataset is (almost) $k$-nested if the set of columns can be partitioned to $k$ parts so that each part is (almost) nested. We consider the algorithmic problems of computing how far a dataset is from being $k$-nested, and for finding a good partition of the columns into $k$ parts. The algorithms are based on spectral partitioning. We apply the methods to real data from ecology and from other applications, and demonstrate the usefulness of the concept.

The rest of this paper is organized as follows. In Section 2 we give the definitions of almost nestedness and segmented nestedness. Section 3 gives the algorithms, and Section 4 the empirical results. Section 5 discusses related work, and Section 6 is a short conclusion.

\section{PROBLEM DEFINITION}

\subsection{Nestedness}

Consider an $n \times m$ 0-1 matrix $M$. In an ecological application, the rows could correspond to sites and columns to species. In a course enrollment data, the rows would correspond to students and the columns to courses. We denote the $i$ th row of $M$ by $M_{i}$ and the $j$ th column of $M$ by $M^{j}$. The rows and the columns of matrix $M$ have also set interpretations. That is, $M_{i}$, except for being a $0-1$ vector, is also used to denote the subset of the species that appear in the $i$-th site. We use vector and set interpretation of the rows and columns of $M$ interchangeably.

Definition 1. An $n \times m$ 0-1 matrix $M$ is fully nested if for any two rows $i$ and $j$ we have $M_{i} \cap M_{j} \in\left\{M_{i}, M_{j}\right\}$.

That is, any two rows of a fully nested matrix have a subset-superset relationship. Alternatively, a matrix $M$ is fully nested if there is a permutation of the rows of $M$ such that in the permuted matrix $M^{\pi}, M^{\pi}{ }_{i} \supseteq M^{\pi}{ }_{j}$ for every $i<j$. From the definition above, it is obvious that checking if a matrix is fully nested can be done in polynomial time.

In practice, the input matrices are not expected to be fully nested. Therefore, we need to define a measure of how far a 0-1 matrix is from being fully nested. We do so by looking at the minimum number of 0 s that need to be transformed into 1 s so that the matrix $M$ becomes nested and we denote this by $\mathcal{N}(M)$. Therefore, for a fully nested matrix $\mathcal{N}(M)=0$. We loosely call matrices with non-zero, but small values of $\mathcal{N}$, almost nested. Next, we formally define the corresponding optimization problem that we call the Minimum Nestedness Augmentation (or Mna).

Problem 1 (Minimum Nestedness Augmentation). Given a 0-1 matrix $M$, find the minimum number of $0 s$ that have to be transformed into $1 \mathrm{~s}$ so that $M$ becomes fully nested.

\section{We have the following.}

Proposition 1. The Minimum Nestedness AugmenTATION problem is NP-complete.

The hardness proof of the problem is easy if we consider the graph-theoretic interpretation of the MNA problem. The input matrix $M$ defines a bipartite graph $G(M)=(B, S, E)$. Every node $b \in B$ corresponds to a row in $M$ and every node $s \in S$ corresponds to a column in $M$. There exists an undirected edge between $b$ and $s$ if and only if $M(b, s)=1$. Therefore, $|B|=n,|S|=m$ and $|E|$ is the number of 1 s in $M$.

A bipartite graph $(B, S, E)$ is a chain graph [26] if there is a bijection $\pi:\{1, \ldots,|B|\} \rightarrow B$ (an ordering of $B$ ) such that $\Gamma(\pi(1)) \supseteq \Gamma(\pi(2)) \supseteq \ldots \supseteq \Gamma(\pi(|B|))$, where $\Gamma$ is a function that maps a node to its neighbors. 
The Minimum Chain Completion problem is as follows. Given a bipartite graph $G(M)$, find the minimum set of edges $F$ that need to be added in $G(M)$ such that the bipartite graph $(B, S, E \cup F)$ is a chain graph. This problem is NP-hard [26]. It is easy to see that the Minimum Chain Completion problem is equivalent the Minimum NestedNess Augmentation problem, and hence MnA is also NPhard. It is trivially in NP.

The equivalence of the matrix and graph formulations also implies the following result.

Proposition 2. For any matrix $M$ we have $\mathcal{N}(M)=$ $\mathcal{N}\left(M^{T}\right)$, where $M^{T}$ denotes the transpose of $M$.

Lemma 1 in [26] states that a bipartite graph is a chain graph if and only if it does not contain a pair of edges that do not share any endpoints (independent edges). We can restate this lemma in terms of the $0-1$ matrix $M$ as follows.

Lemma 1. A 0-1 matrix $M$ is fully nested if and only if it does not contain any submatrix of the form

$$
\left(\begin{array}{ccccc} 
& \vdots & & \vdots & \\
\ldots & 0 & \ldots & 1 & \ldots \\
& \vdots & & \vdots & \\
\ldots & 1 & \ldots & 0 & \ldots \\
& \vdots & & \vdots &
\end{array}\right)
$$

We call such submatrices switch boxes. We show in Section 3 how we use Lemma 1 to design a greedy algorithm for MNA.

Note that MNA considers only transformations of 0s into $1 \mathrm{~s}$, and charges for each such transformation. The reason for this is that in the paleontological/ecological datasets there is high certainty associated with 1s and low certainty associated with 0s. In terms of problem definitions, we can generalize MNA so that we allow changes of $1 \mathrm{~s}$ into $0 \mathrm{~s}$ as well. We call this generalized version of MNA, BIDIRECTIONAL MNA problem (or BMNA), and we formally define it as follows.

Problem 2 (BMna). Given a 0-1 matrix $M$, find the minimum number of 0 s or $1 \mathrm{~s}$ that have to be transformed into $1 s$ or 0 , respectively, so that $M$ becomes fully nested.

We denote by $\mathcal{B}(M)$ the cost of the optimal solution of the BMnA problem for input matrix $M$. The complexity of the BMnA problem is unknown (see [17]). Although the focus of the paper is on the MNA problem, we also show how algorithms for MNA can be adopted to solve the BMNA problem as well. It is rather easy to see the following straightforward relationship between the optimal solutions to the MNA and BMnA problems.

\section{Proposition 3. For a 0-1 matrix $M$ we have}

$$
\mathcal{N}(M) \geq \mathcal{B}(M)
$$

\subsection{Segmented nestedness}

In this subsection we consider the problem of finding a good partition of the columns of a matrix so that the projections of the dataset to the parts are (almost) nested.

Consider the 0-1 matrix shown in Figure 2. Clearly, this matrix is not almost nested. However, there are evidently two almost nested submatrices that are induced by the first and the last 20 columns of the input matrix.

As mentioned in the introduction, such matrices motivate the definition of the segmented version of the MNA problem. ${ }^{2}$ In the segmented version of the problem we wish to partition columns of the matrix into $k$ parts so that the submatrices induced by each part are fully or almost nested. If $\left\{P_{1}, \ldots, P_{k}\right\}$ is a partition of the columns of $M$ into $k$ parts, we denote by $M\left[P_{i}\right]$ the matrix of size $n \times\left|P_{i}\right|$ obtained from $M$ by just considering the columns in $P_{i}$. We can extend the definitions of the fully and almost nested matrices for segmented nestedness.

Definition 2. An $n \times m$ 0-1 matrix $M$ is fully $k$-nested if there is a partition of its columns into $k$ parts $\left\{P_{1}, \ldots, P_{k}\right\}$ such that each $M\left[P_{i}\right]$ is fully nested.

An immediate consequence of the above definition is the following.

Proposition 4. For integers $k_{2}, k_{1}$ with $k_{2}>k_{1}$, if a $0-1$ matrix $M$ is fully $k_{1}$-nested, then it is also fully $k_{2}$-nested.

The matrices that appear in practice are not expected to be fully $k$-nested, but rather almost $k$-nested. That is, the submatrices $M\left[P_{i}\right]$ are almost nested.

For input matrix $M$, the segmented version of the MNA problem asks for a partition $\left\{P_{1}, \ldots, P_{k}\right\}$ of the columns of $M$ such that the total number of conversions of 0 s to 1 s in the $M\left[P_{i}\right]$ 's is minimized. We call this problem the $k$-MNA problem and we formally define it as follows.

Problem 3 ( $k$-MnA). Given a $0-1$ matrix $M$ and an integer $k$, find a partition of the columns of $M$ into $k$ parts $\left\{P_{1}, \ldots, P_{k}\right\}$, such that

$$
\mathcal{N}_{k}(M)=\sum_{i=1}^{k} \mathcal{N}\left(M\left[P_{i}\right]\right)
$$

is minimized.

Therefore, fully $k$-nested datasets have $\mathcal{N}_{k}(M)=0$, while almost $k$-nested datasets have small values of $\mathcal{N}_{k}(M)$. Figure 2 is an example of a dataset $M$ that has high value of $\mathcal{N}(M)$ but a small value of $\mathcal{N}_{2}(M)$.

Proposition 5. For a 0-1 matrix $M$ and integers $k_{1}, k_{2}$ such that $k_{2}>k_{1}$ we have $\mathcal{N}_{k_{1}}(M) \geq \mathcal{N}_{k_{2}}(M)$.

Since the $k$-MnA is a generalization of the MNA problem, $k$-MNA is also NP-hard.

Instead of looking for a partition of the columns that defines nested submatrices one can alternatively define the problem of removing the minimum number of rows and columns from the input matrix, so that the remaining (maximal) submatrix is almost nested. By using the results of [25] one can show that this variation of the segmented nestedness problem is also NP-hard. In the case where we are restricted in removing only rows or only columns of the input matrix, the problem becomes solvable in polynomial time. The relationship between these alternatives and the $k$-MNA problem is the following: while $k$-MNA looks for groups of columns (or rows) so that there is a nesting structure within each group,

\footnotetext{
${ }^{2}$ The term segmented comes from the definition of "Segmentation Problems" given in [15].
} 
the aforementioned variants look for removal of columns and rows so that a nesting structure appears. Therefore, if one thinks of the $k$-MNA problem as an analogue for clustering, then the above alternatives correspond to problems like outlier detection or finding a single cluster.

We will also refer to the segmented version of Problem 2 as the $k$-BMnA problem. The problem definition and the corresponding properties are in accordance to those of Problem 3 and thus omitted. For input matrix $M$, we use $\mathcal{B}_{k}(M)$ to denote the optimal solution of the $k$-BMNA problem for $M$.

\section{ALGORITHMS}

\subsection{Algorithms for estimating nestedness}

In this section we consider algorithms for estimating $\mathcal{N}(M)$ for a matrix $M$. As the task is NP-hard, we cannot hope for an exact solution; rather, we will compute scores $\widehat{\mathcal{N}}(M)$ that are upper bounds for $\mathcal{N}(M)$.

Two simple heuristics have been used in the ecology literature for the nestedness and related problems [6, 5]. The first algorithm is RowSum. For input matrix $M$, the algorithm counts the number of $1 \mathrm{~s}$ in each row and reorders the rows of $M$ in decreasing order of their row sums. The algorithm then converts the necessary $0 \mathrm{~s}$ to $1 \mathrm{~s}$ so that the final matrix becomes fully nested. The complexity of the algorithm is $O(m n+n \log n)$. The following example shows the cost of the solution output by the RowSum algorithm can be arbitrarily bad compared to the cost of the optimal solution.

EXAmPle 1. Consider the $(p+2) \times(3 m)$ matrix $M$ that has the following structure

$$
M=\left(\begin{array}{ccccccccc}
0 & \ldots & 0 & 1 & \ldots & 1 & 1 & \ldots & 1 \\
1 & \ldots & 1 & 1 & \ldots & 1 & 0 & \ldots & 0 \\
\vdots & \vdots & \vdots & \vdots & \vdots & \vdots & \vdots & \vdots & \vdots \\
1 & \ldots & 1 & 1 & \ldots & 1 & 0 & \ldots & 0 \\
1 & \ldots & 1 & 0 & \ldots & 0 & 1 & \ldots & 1
\end{array}\right)
$$

The RowSum algorithm can keep the order of the rows of $M$ unchanged, causing a cost of $2 m+m p$. The cost of the optimal solution would be just $2 m$, achieved by moving the last row of $M$ on top. Therefore, the performance ration of RowSum is $\frac{2 m}{2 m+m p}$, which goes to 0 as $p$ grows.

Some straightforward alternatives to RowSum can be the ColSum and the BestSum algorithms. The Colsum is exactly the same as the RowSum but it operates on the columns of $M$. That is, the columns of $M$ are rearranged in decreasing order of their column sums and the necessary 0s are converted into 1s so that the final matrix is fully nested. For given input matrix $M$, the BestSum algorithm is the best of RowSum and Colsum for the given input. Both these algorithms can give solutions with cost arbitrarily bad with respect to the optimal with input the matrix given in Example 1.

Some other algorithms for quantifying nestedness have also been considered in the ecological literature (see, e.g., $[23,9,21])$, but they are fairly heuristic and difficult to analyze.

In the rest of the section we give a simple greedy algorithm for the MNA problem. We also show how we can adopt this algorithm to solve the BMNA problem as well.
Consider an input matrix $M$ of size $n \times m$. By Lemma 1 , $M$ is fully nested if it does not contain any switch boxes. Given this, we can design a greedy algorithm that switches 0 s into $1 \mathrm{~s}$ one at a time. At every step, the algorithm converts the 0 that participates in the largest number of distinct switch boxes. Consider entry $(i, j)$ of the matrix. The coverage $C(i, j)$ of entry $(i, j)$ is the number of switch boxes this entry participates in. By converting the value in $(i, j)$ from 0 to 1 , all the $C(i, j)$ switch boxes are resolved.

After each conversion, the algorithm recalculates the coverages of the remaining $0 \mathrm{~s}$ in the matrix and proceeds in the next greedy step. Algorithm 1 gives an outline of the Greedy algorithm.

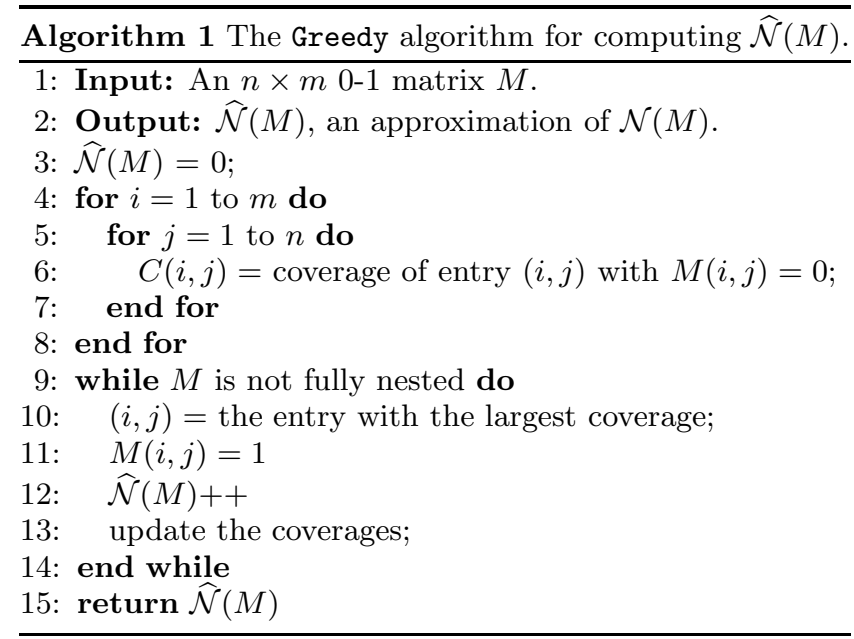

The running time of the Greedy algorithm is $O(m n+$ $\left.I T_{1} T_{2}\right)$, where $I$ is the number of iterations of the while loop, $T_{1}$ the time required for finding the maximum coverage, and $T_{2}$ the time required for updating the coverages.

By using a heap, $T_{1}$ is $O(1)$ and $T_{2}$ is $O(m n \log (m n))$. When entry $(i, j)$ is converted from 0 to 1 , we only have to make the following updates: (i) decrease by 1 the coverages of entries $(v, u)$ with $M_{v u}=0, M_{i u}=1$, and $M_{v j}=1$; (ii) increase by 1 the coverages of entries $(i, u)$ and $(v, j)$ with $M_{v u}=1, M_{i u}=0$, and $M_{v j}=0$. These are due to (i) switch boxes that $(i, j)$ participated in and that are removed, and (ii) switch boxes that are created by the conversion.

As the maximum number of iterations is $O(m n)$, the total time is $O\left((m n)^{2} \log (m n)\right)$. In practice the number of iterations is much smaller than $O(m n)$, indicating that the method scales reasonably well.

The same greedy principle can be used for solving the BMnA problem. The corresponding Bi-Greedy algorithm is essentially the same as the Greedy algorithm above, with the only difference that since both 0 s are transformed into $1 \mathrm{~s}$ and $1 \mathrm{~s}$ into $0 \mathrm{~s}$, the coverages of both 0 and 1 entries are computed in the beginning and updated in every iteration. Whenever entry $(i, j)$ is converted from 0 to 1 the following updates need to be done by the Bi-Greedy algorithm: (i) decrease by 1 the coverages of entries $(v, u)$ with $M_{v u}=0$, $M_{i u}=1$, and $M_{v j}=1$; (ii) increase by 1 the coverages of entries $(v, u)$ with $M_{v u}=1, M_{i u}=0$, and $M_{v j}=0$; (iii) increase by 1 the coverages of entries $(i, u)$ and $(v, j)$ with $M_{v u}=1, M_{i u}=0$, and $M_{v j}=0$; (iv) decrease by 1 the coverages of entries $(i, u)$ and $(v, j)$ with $M_{v u}=0, M_{i u}=1$, and $M_{v j}=1$. The complementary updates are made when 
entry $(i, j)$ is converted from 1 to 0 . The running time of the Bi-Greedy algorithm is of the same order as the running time of the Greedy algorithm discussed above. Note that the algorithm is designed so that at most one conversion is allowed per entry.

\subsection{Algorithms for estimating segmented nestedness}

In this section we give algorithms for the $k$-MNA problem. The algorithms for the $k$-BMNA problem are easily developed using the same principles, and thus their discussion is omitted. Remember that the task is to find a partition $\left\{P_{1}, \ldots, P_{k}\right\}$ of the columns of the matrix $M$ so that the projections $M\left[P_{i}\right]$ of $M$ to these columns have a small value of $\mathcal{N}(M)$. Again, as the problem is NP-hard, we have to be content with producing upper bounds $\widehat{\mathcal{N}}_{k}(M)$ for $\mathcal{N}_{k}(M)$.

We approach the problem as a clustering problem for the columns of the input matrix $M$. When should two columns be placed to the same group in the partition? Obviously, if the columns $a$ and $b$ are similar, i.e., they tend to have $1 \mathrm{~s}$ in the same rows, then it makes sense to have them in the same part $P_{i}$. On the other hand, if $a$ and $b$ are independent or negatively correlated, then they should be in different parts of the partition.

This simple observation leads to an algorithm for computing an approximation to $\mathcal{N}_{2}(M)$ : define a similarity notion between columns, use a spectral bisection method [22] to obtain the two parts of the partition, and then compute the score for both parts using the Greedy algorithm. By recursive applications of the algorithm we obtain a way of approximating $\mathcal{N}_{k}(M)$ as well.

We first define two simple and intuitive similarity functions between the columns of the input matrix $M$. Then, we combine these functions with a simple spectral bisection algorithm to solve the 2-MNA problem.

One straightforward measure of similarity that serves our purposes is the correlation similarity. We define the correlation similarity between two columns $M^{a}$ and $M^{b}$ as

$$
\operatorname{CorrS}\left(M^{a}, M^{b}\right)=1+\rho_{a b},
$$

where $\rho_{a b}$ is the Pearson correlation between columns $a$ and $b$. As $\rho_{a b}$ takes values in the range $[-1,1]$, CorrS takes values in $[0,2]$. Value 2 is obtained if the columns are identical, and value 0 when they are perfectly anticorrelated.

Alternatively, we use the following inclusion similarity between columns $M^{a}$ and $M^{b}$ :

$$
\operatorname{InclS}\left(M^{a}, M^{b}\right)=\frac{M^{a} \cap M^{b}}{\min \left\{\left|M^{a}\right|,\left|M^{b}\right|\right\}} .
$$

The inclusion similarity captures our intuition that two columns need to be in the same cluster if the one includes the other. For example, when column $a$ is included in column $b$ (or vice versa), then $\operatorname{InclS}\left(M^{a}, M^{b}\right)=1$, and thus columns $a$ and $b$ are considered very similar. Similarly, the inclusion similarity of two non-intersecting columns is zero.

Spectral algorithms are important tools for a wide range of problems such as, solving linear systems [20], ordering problems [1, 16], data clustering [18, 10] and many more. Before describing the spectral bisection algorithm we use, we give some background for the spectral method in general.

Consider a weighted undirected graph $G=(V, E)$ where each $(i, j) \in E$ has a weight $w_{i j}$. Let $A$ be the matrix with $A(i, j)=w_{i j}$ if $(i, j) \in E$ and $A(i, j)=0$ otherwise. The
Laplacian of $A$ is defined to be the symmetric and zero-sum matrix $\mathcal{L}=D-A$, where $D$ is a diagonal matrix whose $(i, i)$-th entry is $d_{i}=\sum_{(i, j) \in E} w_{i j}$. The eigenvalues of $\mathcal{L}$ are real and nonnegative, and the smallest eigenvalue is 0 (corresponding to the eigenvector of all 1s). The eigenvector $v$ that corresponds to the second smallest eigenvalue of $\mathcal{L}$ is also known as the Fiedler vector.

We consider the graph $A$, whose nodes are the columns of the input matrix $M$. There is an edge between all pairs of nodes, and the weight of the edge $(a, b)$ is $\operatorname{CorrS}\left(M^{a}, M^{b}\right)$ (Equation (1)) or $\operatorname{InclS}\left(M^{a}, M^{b}\right.$ ) (Equation (2)). We form the Laplacian matrix $\mathcal{L}_{A}$ of the graph $A$ and compute the Fiedler vector $v$. Then $v$ is a vector with $m$ components.

Each element $v_{a}$ of $v$ defines a partition by $P_{1}=\left\{b \mid v_{b} \leq\right.$ $\left.v_{a}\right\}$ and $P_{2}=\left\{b \mid v_{b}>v_{a}\right\}$. For each $v_{a}$, we evaluate $\widehat{\mathcal{N}}\left(M\left[P_{1}\right]\right)+\widehat{\mathcal{N}}\left(M\left[P_{2}\right]\right)$ and return the partition with the smallest value of the sum.

The algorithm can be augmented by local moves. That is, we start from the initial partition given by the spectral bisection method, and then repeatedly search for columns that can be moved from one part to the other so that the score improves.

If $k>2$, then we recursively call the algorithm on each of the parts to check which one should be divided further. The method, called Partition, is described also in Algorithm 2.

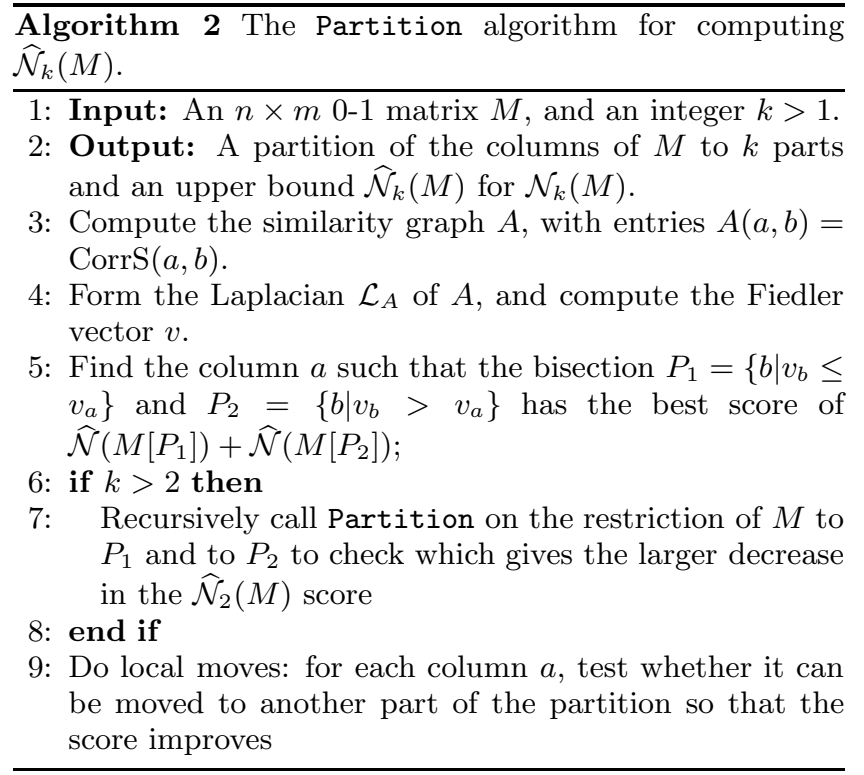

Every call of the Partition algorithm requires time $O\left(T_{\lambda}+m T_{p}\right)$, where $T_{\lambda}$ is the time needed for the eigenvalue computation and $T_{p}$ is the time required for the approximation of $\mathcal{N}$ at step 5 of the algorithm. The dominating factor is the computation of $\widehat{\mathcal{N}}$ for $k=1$ for both sets of $m$ different partitions, resulting a total time complexity of $O\left(m^{3} n^{2} \log (m n)\right)$. In practice the method scales to datasets of moderate size, as the above bound is a worst-case one.

We will compare the performance of the Partition algorithm with the CorrS and InclS similarity functions with two straightforward heuristics, the kmeans and the InclS-kmeans. The kmeans algorithm performs a clustering of the columns considering each column as an observation consisting of $n$ attributes (where $n$ is the number of rows of the input matrix). We use the Hamming distance func- 
tion as the optimization criterion for the kmeans algorithm. The InclS-kmeans again clusters the columns of the input matrix, this time using as input the similarity matrix induced by the InclS function. In this case, the centers of the $\mathrm{k}$-means procedure are bound to be from the input points, since only the similarity matrix is given as input.

\subsection{Selecting the value of $k$}

The concept of $k$-MNA has the parameter $k$ that can vary. For any dataset $M$, we have $\mathcal{N}_{k}(M) \geq \mathcal{N}_{k+1}(M)$, as the ability to divide the columns into $k+1$ sets instead of $k$ sets will make it easier to have small number of nestedness violations. At the limit, when $k=m$, the number of columns in $M$, we have $\mathcal{N}_{m}(M)=0$. So how should a good value of $k$ be chosen?

This is, of course, a classical example of the model selection problem. Different solutions abound: one can use MDL, BIC, AIC, cross-validation, etc. (see, e.g., [12] for a description of some of the methods).

Here we use a fairly simple alternative: we monitor the change of the score $\widehat{\mathcal{N}}_{k}(M)$ as a function of $k$; when the score flattens, the correct value of $k$ is reached. This, of course, is a heuristic approach, but seems to be sufficient. We also compare the change in the score with the change in the score $\widehat{\mathcal{N}}_{k}\left(M^{\pi}\right)$, where $M^{\pi}$ is a dataset obtained from $M$ by permuting each column of $M$ independently at random. ${ }^{3}$ That is, we test whether the score on real data drops clearly faster than for random data with the same density. Other methods of selecting $k$ are left for further study.

\section{EXPERIMENTS}

In this section, we give experimental evidence of the utility of the nestedness concept. For this, we use both synthetic and real datasets; the latter come from a variety of application domains like ecology, paleontology and students' course enrollment data.

\subsection{Synthetic datasets}

To test the behavior of the algorithms we generated synthetic data as follows. For given $n$ and $m$ we first generate a fully nested 0-1 matrix by first sampling row counts $r_{i}$ uniformly between 0 and $m$, and sorting them so that $r_{i} \geq r_{j}$ for every $i<j$. Then we generate rows so that row $i$ has $1 \mathrm{~s}$ in its first $r_{i}$ columns and $0 \mathrm{~s}$ in the other columns. Let such a matrix be $H$, and let $H^{1}$ be all the cells in $H$ that have value 1 and $H^{0}$ all the cells that have value 0 . Given $H$ we generate our synthetic matrices by altering entries of $H^{1}$ from 1 to 0 , and entries of $H^{0}$ from 0 to 1 . An entry in $H^{1}$ keeps its original value with probability $1-p$, and it is converted to 0 with probability $p$. Similarly, the entries in $H^{0}$ are switched to 1 with probability $q$ and they maintain their original value with probability $1-q$. Thus, $p$ is the probability of an 0 -entry in $H^{1}$ and $q$ the probability of an 1-entry in $H^{0}$. The interesting parameter values are ones where $p>q$ and $q$ is fairly close to 0 ; this is due to the asymmetry of 0 s and $1 \mathrm{~s}$ mentioned earlier. Figure 3 shows the relative performance of the three algorithms Greedy, RowSum and ColSum algorithms for synthetic datasets generated as above. Note that when $p=0$ and $q=0$, the generated

\footnotetext{
${ }^{3}$ Note that in a fully nested dataset there are no switch boxes; thus we cannot use the swap randomization method [13].
}

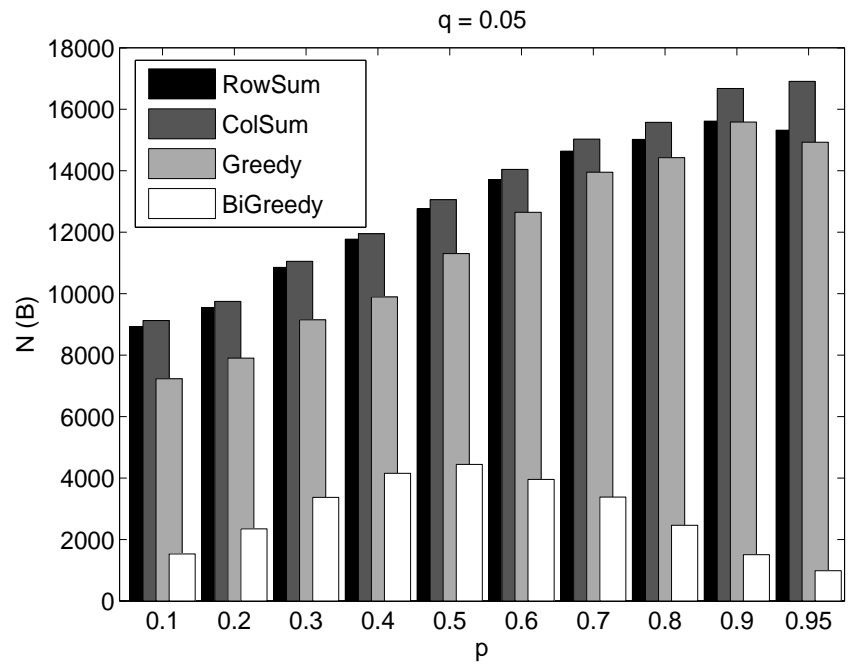

Figure 3: Comparative performance of algorithms for MNA problem on synthetic datasets for $q=0.05$. x-axis: parameter $p$; y-axis: the quantity $\widehat{\mathcal{N}}(M)$ of the input $M$ for RowSum, ColSum and Greedy algorithm and the quantity $\widehat{\mathcal{B}}(M)$ for Bi-Greedy algorithm.

matrices are fully nested and all algorithms find the optimal solution that has cost 0 . In Figure 3 we compare the number of flips done by the different algorithms for datasets generated with $q=0.05$ and $p \in[0.1,0.95]$. Among these three algorithms we can clearly see that the Greedy algorithm consistently outperforms the other two, and the difference is fairly high especially for smaller values of $p$. In the same figure we additionally report the cost of the Bi-Greedy algorithm using cost function $\mathcal{B}$ instead of $\mathcal{N}$. Note that although the results of $\mathrm{Bi}-\mathrm{Greedy}$ are not comparable with the results of the other algorithms, they are given here for completeness.

\subsection{Habitat datasets}

We have also tested the relative performance of the different algorithms for MNA by using a set of real datasets available by AICS Research Inc, University Park, New Mexico, and The Field Museum, Chicago. The datasets are available online ${ }^{4}$ and they have been used for a wide range of ecological studies [3, 9, 7, 2, 23]. The collection contains 280 datasets in the form of presence/absence matrices, that cover a wide variety of taxa (mammals, bats, land and freshwater birds, reptiles and amphibians, fish, terrestrial arthropods, terrestrial mollusks, plants and other miscellaneous species). Figure 4 serves as a summary of comparisons between the Greedy algorithm and the best of the RowSum and ColSum algorithms (BestSum). For the purposes of the experiment, we group the datasets with respect to their densities (number of 1's in the matrix divided by the size of the matrix). For each density range, we count the number of datasets in the range for which Greedy performs better, equal or worse than BestSum algorithm. For most datasets with small density the Greedy algorithm performs considerably better than BestSum, and throughout the datasets Greedy is only seldom worse than BestSum. Figure 5 shows

\footnotetext{
${ }^{4}$ http://www. aics-research.com/nestedness/
} 


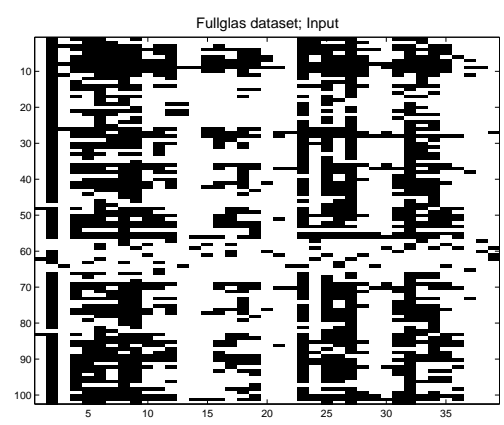

(a) Fulglas dataset; Input matrix

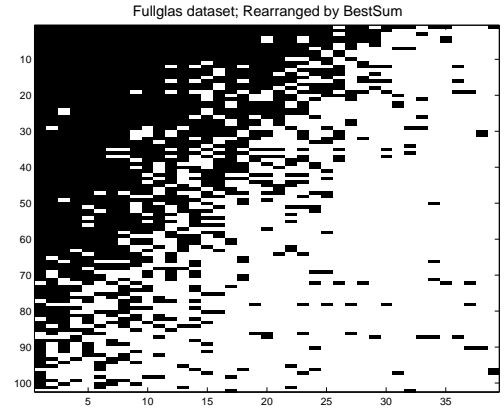

(b) Fulglas dataset; Rearrangement by BestSum

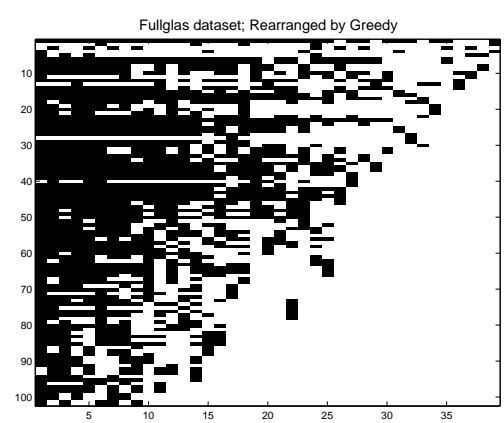

(c) Fulglas dataset; Rearrangement by Greedy

Figure 5: Rearrangements of the Fulglas dataset matrix

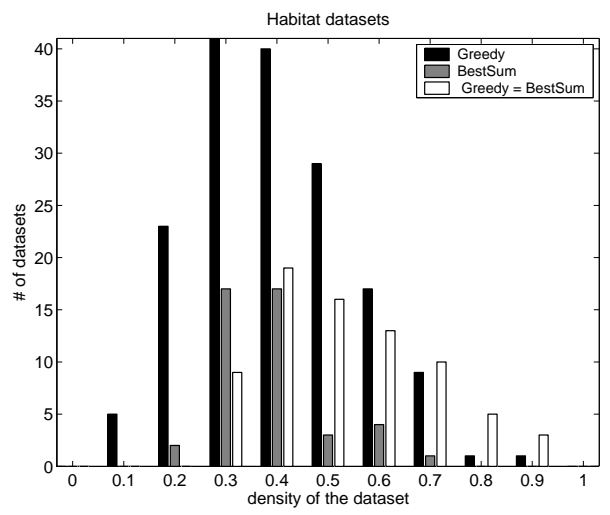

Figure 4: The performance of the algorithms on the habitats datasets. The columns show the number of datasets for which the Greedy algorithm or the BestSum algorithm (the better of RowSum and ColSum) gives better results or whenever they are equal.

an indicative example of this performance for the Fullglas dataset. The dataset has 102 rows, 39 columns and 1503 non-zero entries. Figure 5(a) renders the input matrix. The matrix corresponds to presence/absence information of prairie plants (goldenrods, milkweeds, and legumes) in 102 prairie fragments in Iowa and Minnesota. The BestSum algorithm makes 2026 conversions, while the Greedy only needs 959. The rearranged matrices output by the two algorithms are shown in Figures 5(b) and 5(c). The example above illustrates the philosophy of the Greedy algorithm. Instead of a rearrangement that brings rows with larger row sum before rows with smaller row sum, it rather prefers bringing some sparse rows on the top for the sake of creating a matrix with a clear triangular form. Notice that the rearranged matrices output by the Greedy algorithm have no 1s in their right lower part.

\subsection{Segmented nestedness}

In this section we test the algorithms for computing $\widehat{\mathcal{N}}_{k}(M)$. There are two problems to consider: whether the algorithm is able to recognize the cases where the dataset has a clear $k$-nested structure, and whether there are clear cases of $k$-nested structure in real data. We evaluate the algorithms for approximating $\mathcal{N}_{k}(M)$ via experiments on both synthetic and real datasets. We generate the synthetic datasets using the same method described in Section 4.1. For given $m$ and $n$, we use the method described in Section 4.1 to generate two matrices $M_{1}$ and $M_{2}$ of sizes $n \times m_{1}$ and $n \times m_{2}$, such that $m_{1}+m_{2}=m$. By random permutations of the rows and the columns of $M_{1}$ and $M_{2}$, we obtain the permuted matrices $M_{1}^{\prime}$ and $M_{2}^{\prime}$. The final matrix $M$ is obtained by concatenating the $i$-th row of $M_{1}^{\prime}$ with the $i$-th row of $M_{2}^{\prime}$. The matrices generated in such a way are almost 2-nested, i.e., they have a small value of $\mathcal{N}_{2}(M)$. We use such matrices as input to the bisection algorithms we described in Section 3.2. The same technique can be used to generate almost $k$-nested datasets for any $k$. Figure 6 shows the performance of the Partition algorithm using the CorrS and InclS similarity functions and the effect of the local moves. We compare the performance of these algorithms with the performance of two straightforward heuristics, the kmeans and the InclS-kmeans (see Section 3.2). As a baseline, we also compare the quantity $\widehat{\mathcal{N}}_{2}$ returned by the algorithms to the ground truth, i.e., the value determined by the generation process. As shown in Figure 6 the Partition algorithm for both the similarity measures defined in Section 3.2 outperforms the other heuristics. This performance is independent of how balanced the sizes of the planted nests are (Figure 6(a)) or the noise level of the dataset (Figure 6(b)). The cost achieved by the Partition algorithm is quite close to the ground truth. The effect of the local moves step becomes more obvious for datasets generated to have components of unequal sizes. In many cases, the $\widehat{\mathcal{N}}_{k}$ score of the Partition algorithm with local moves and the Incls similarity function is better than the score obtained by the ground truth partition.

The next experiment investigates the selection of the correct value of $k$. We generated datasets that have 1,2 , or 3 -nested structure, and tested the values of $\widehat{\mathcal{N}}_{k}$ and $\widehat{\mathcal{B}}_{k}$ for $k=1,2,3,4,5$. Table 1 shows the results, together with the results for the permuted version of the datasets. We observe that for 1-nested data the score $\widehat{\mathcal{N}}_{k}$ stays about the same for all values of $k$, while for 2-nested data the score $\widehat{\mathcal{N}}_{k}(M)$ drops a lot when moving from $k=1$ to $k=2$, and for 3-nested data the score $\widehat{\mathcal{N}}_{k}(M)$ drops a lot when moving from $k=1$ to $k=2$ and $k=3$. I.e., the drop rate can be used as an indication of the correct value of $k$. The same 

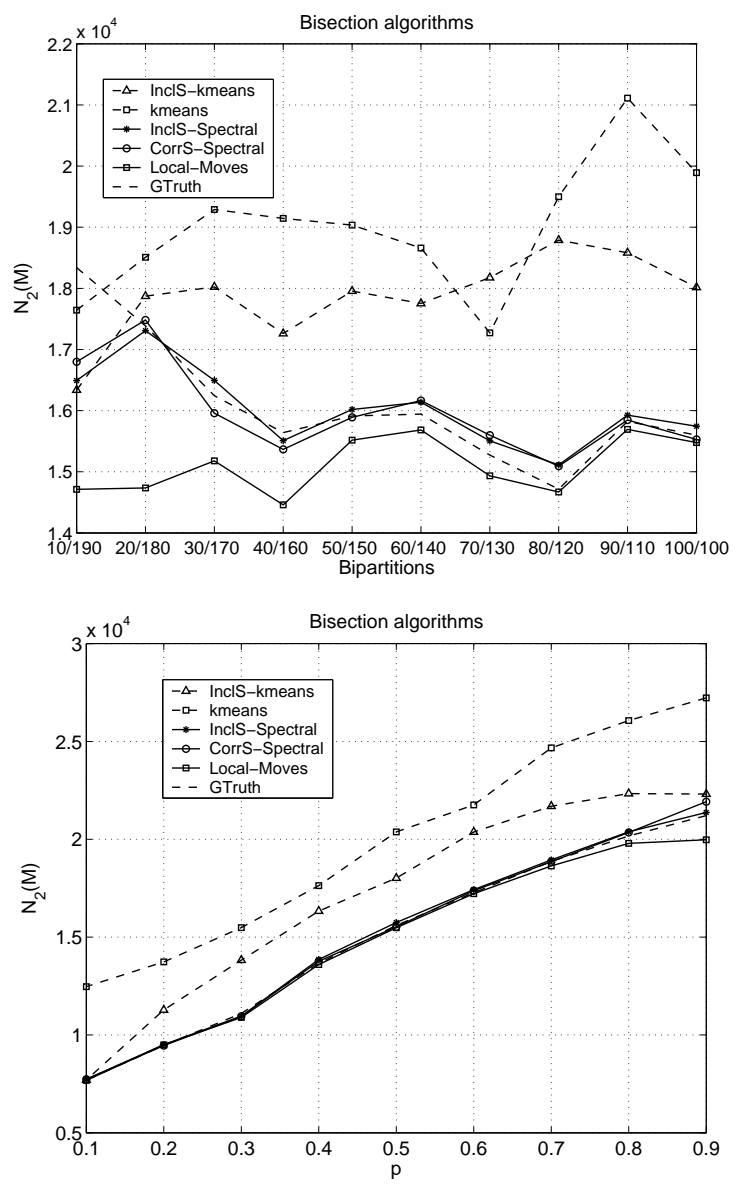

Figure 6: Comparative performance of bisection algorithms for approximating $\mathcal{N}_{2}$. Upper panel: Data set generated with $p=0.5$ and $q=0.01$ (see text), with two matrices of 200 rows and varying number columns in two nested parts. $x$-axis: the sizes of the nested parts; y-axis: $\widehat{\mathcal{N}}_{2}$. Lower panel: Data set generated with $q=0.01$ and varying $p ; 200$ rows, and two nested parts of 100 columns. x-axis: the value of $p$; y-axis: $\widehat{\mathcal{N}}_{2}$. Algorithms: kmeans and the InclS-kmeans: see Section 3.2; InclS-Spectral and CorrS-Spectral: the Partition algorithm with the InclS and CorrS similarity metrics, but without local moves; Local-Moves: the Partition algorithm with the Incls similarity metric and with local moves; GTruth: the ground truth from the data generation process. patterns are observed even more clearly for the values of $\widehat{\mathcal{B}}_{k}$. (The drop in the score of the permuted datasets tells us something about how large changes should be expected on the average.) Table 2 shows the same type of results

\begin{tabular}{|rrrr|rrr|}
\hline \multicolumn{7}{|c|}{ true $\mathrm{k}=1$} \\
\hline$k$ & $\widehat{\mathcal{N}}_{k}(M)$ & $\widehat{\mathcal{N}}_{k}\left(M^{\pi}\right)$ & std & $\widehat{\mathcal{B}}_{k}(M)$ & $\widehat{\mathcal{B}}_{k}\left(M^{\pi}\right)$ & std \\
\hline 1 & 6643 & 18717 & 67 & 2194 & 9861 & 25 \\
2 & 5263 & 18523 & 45 & 2170 & 9810 & 54 \\
3 & 4202 & 17383 & 330 & 2156 & 9741 & 40 \\
4 & 3944 & 16731 & 587 & 2139 & 9661 & 53 \\
5 & 3789 & 15954 & 354 & 2121 & 9561 & 59 \\
\hline \multicolumn{7}{|c|}{ true k=2 } \\
\hline$k$ & $\widehat{\mathcal{N}}_{k}(M)$ & $\widehat{\mathcal{N}}_{k}\left(M^{\pi}\right)$ & std & $\widehat{\mathcal{B}}_{k}(M)$ & $\widehat{\mathcal{B}}_{k}\left(M^{\pi}\right)$ & std \\
\hline 1 & 12790 & 18107 & 51 & 6021 & 10162 & 55 \\
2 & 7163 & 17814 & 68 & 2212 & 10134 & 46 \\
3 & 6270 & 17395 & 246 & 2200 & 10079 & 12 \\
4 & 5744 & 16037 & 92 & 2186 & 10023 & 59 \\
5 & 5481 & 15353 & 225 & 2166 & 9906 & 51 \\
\hline \multicolumn{7}{|c|}{ true k=3 } \\
\hline$k$ & $\widehat{\mathcal{N}}_{k}(M)$ & $\widehat{\mathcal{N}}_{k}\left(M^{\pi}\right)$ & std & $\widehat{\mathcal{B}}_{k}(M)$ & $\widehat{\mathcal{B}}_{k}\left(M^{\pi}\right)$ & std \\
\hline 1 & 14622 & 18768 & 73 & 7405 & 10280 & 49 \\
2 & 10442 & 18532 & 56 & 4525 & 10256 & 32 \\
3 & 6579 & 17508 & 194 & 2195 & 10216 & 40 \\
4 & 6248 & 16816 & 524 & 2183 & 10077 & 10 \\
5 & 5842 & 15824 & 44 & 2155 & 10088 & 37 \\
\hline
\end{tabular}

Table 1: The $k$-nestedness count on generated datasets with 1,2 , or 3 components. True $k$ : the number of components in the data; $k$ : the input parameter given to the Partition algorithm; $\widehat{\mathcal{N}}_{k}(M)$ $\left(\widehat{\mathcal{B}}_{k}(M)\right)$ : the MNA (BMNA) score of the partition; $\widehat{\mathcal{N}}_{k}\left(M^{\pi}\right)\left(\widehat{\mathcal{B}}_{k}\left(M^{\pi}\right)\right)$ : the MNA (BMNA) score for the dataset where each column has been permuted independently; std: standard deviation of $\widehat{\mathcal{N}}_{k}\left(M^{\pi}\right)$ and $\widehat{\mathcal{B}}_{k}\left(M^{\pi}\right)$. The size of the generated data is 200 rows and 200 columns. The parameters are $p=0.1$ and $q=0.01$ (see text).

for the paleontological and course datasets. We observe that the drop continues until $k=3$ for the paleontological data and until $k=2$ for the course data, but levels off after that. In all cases the drop is larger for the real dataset than for the permuted one. The interpretation of the results is fairly clear for both real datasets. The paleontological dataset has a 3-nested structure, and the same is true also for the course dataset. In the course dataset there are actually students from two different curricula: the names of the courses have changed, and some students have moved from one system to another. This explains why there are clearly more than one components in the data.

Figure 7 shows the results on the paleontological dataset. Panels (a) and (b) show the results of partitioning the columns into two or three parts so as to maximize nestedness. The dataset is drawn so that the order of the rows corresponds to the temporal order of the sites, and the order of the columns corresponds to the order of the occurrence times of the species. The sets of columns are nicely consecutive in the figure, indicating the expected result that the nested subsets of columns are temporally connected. Panel (c) shows how the subsets of the data projected to 3 parts look like, with the rows reordered so as to show maximum nestedness. We observe a quite strong nested structure in each plot. 


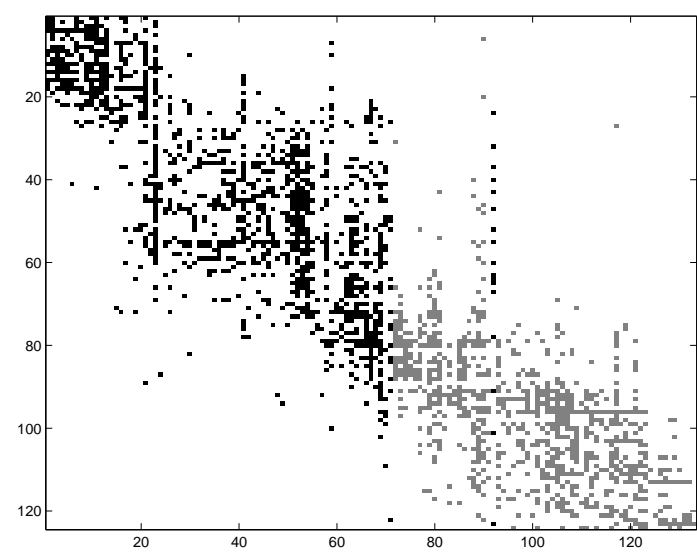

(a) Paleo dataset; 2-partitioning of the columns, with 64 and 75 columns.

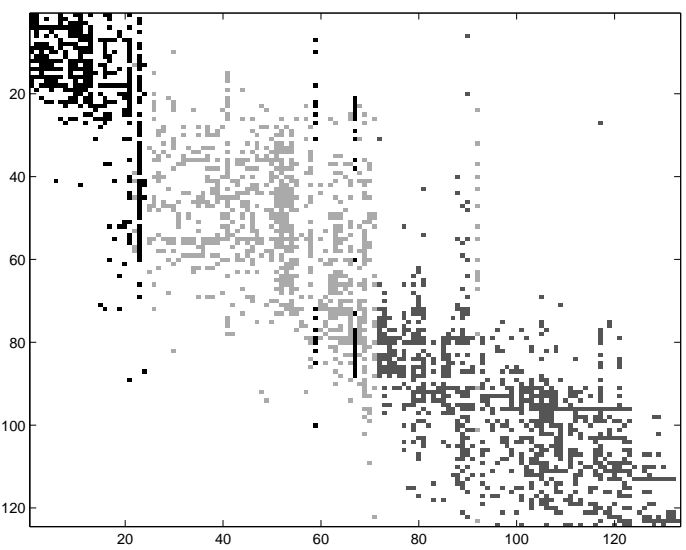

(b) Paleo dataset; 3-partitioning of the columns, with 25,47 , and 61 columns
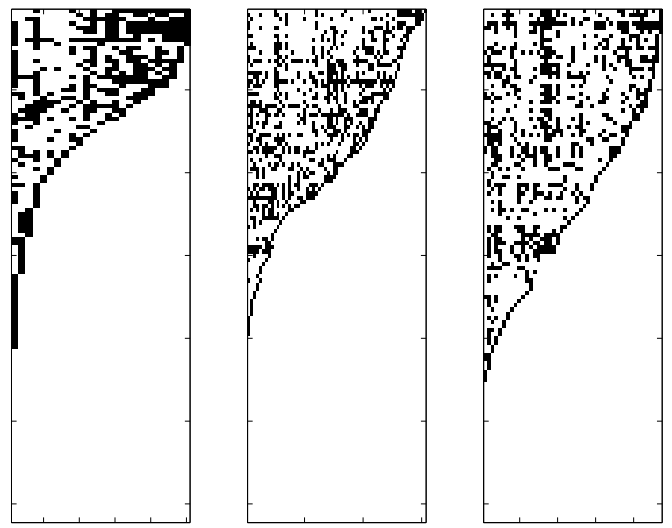

(c) The three subsets of the columns of the paleontological dataset, with rows and columns reordered by the Greedy algorithm.

Figure 7: Segmented nestedness in the paleontological dataset.

\begin{tabular}{|rrrr|rrr|}
\hline \multicolumn{7}{|c|}{ Paleo data } \\
\hline$k$ & $\widehat{\mathcal{N}}_{k}(M)$ & $\widehat{\mathcal{N}}_{k}\left(M^{\pi}\right)$ & std & $\widehat{\mathcal{B}}_{k}(M)$ & $\widehat{\mathcal{B}}_{k}\left(M^{\pi}\right)$ & std \\
\hline 1 & 12156 & 12534 & 92 & 1749 & 1912 & 13 \\
2 & 6375 & 10662 & 66 & 1566 & 1829 & 21 \\
3 & 4674 & 9058 & 163 & 1397 & 1773 & 26 \\
4 & 3933 & 7971 & 73 & 1277 & 1720 & 15 \\
5 & 3505 & 7360 & 295 & 1181 & 1673 & 14 \\
\hline \multicolumn{7}{|c|}{ Course data } \\
\hline$k$ & $\widehat{\mathcal{N}}_{k}(M)$ & $\widehat{\mathcal{N}}_{k}\left(M^{\pi}\right)$ & std & $\widehat{\mathcal{B}}_{k}(M)$ & $\widehat{\mathcal{B}}_{k}\left(M^{\pi}\right)$ & std \\
\hline 1 & 105814 & 140310 & 892 & 20013 & 27676 & 65 \\
2 & 66196 & 126990 & 345 & 16546 & 27069 & 85 \\
3 & 60713 & 109660 & 331 & 14124 & 26831 & 35 \\
4 & 49420 & 93397 & 1140 & 13842 & 26555 & 92 \\
5 & 47485 & 86102 & 1201 & 13503 & 26407 & 109 \\
\hline
\end{tabular}

Table 2: The $k$-nestedness count on real datasets. $k$ : the input parameter given to the Partition algorithm; $\widehat{\mathcal{N}}_{k}(M)\left(\widehat{\mathcal{B}}_{k}(M)\right)$ : the MNA (BMNA) score of the partition; $\widehat{\mathcal{N}}_{k}\left(M^{\pi}\right)\left(\widehat{\mathcal{B}}_{k}\left(M^{\pi}\right)\right)$ : the MNA (BMNA) score for the dataset where each column has been permuted independently; std: standard deviation of $\widehat{\mathcal{N}}_{k}\left(M^{\pi}\right)$ and $\widehat{\mathcal{B}}_{k}\left(M^{\pi}\right)$. The data sizes are paleontological: $124 \times 139$ and course: $2401 \times 106$.

\section{RELATED WORK}

The concept of nestedness has its roots in ecological studies where nested subsets have been observed in presence/absence matrices of species in biotas. The main focus of the ecological studies has been applying the concept to the study of species distribution. In more computationally oriented work, the main effort has been towards defining measures of nestedness and devising randomization tests for checking whether the nestedness score of a given matrix is significant or whether it can appear by chance.

Several indices of nestedness have been proposed like for example the temperature of a matrix [3], the $N_{0}$ index [19, 24] the $N_{1}$ index $[8,24]$, the $N_{C}$ index [24], the $U$ index [8] and lately the discrepancy [5]. Many of these indices are rather informally defined.

Randomization tests have been developed for comparing the nestedness index of a given matrix with that of matrices generated using an underlying generative model. The models used and tested in the ecological literature include those that produce matrices with the same density, or the same row or (or and) column margins [5, 19, 24]. The randomization tests indicate whether the nestedness evaluated using one of the above indices are significant or they could have occurred by chance. The nestedness indices and the randomization methods have been subject to extensive comparisons and have been applied to a wealth of ecological datasets; see [23] for a review.

The study of the computational problems related to the computation of the nestedness indices has been limited. For example, [6] studies the properties of discrepancy for a given family of $0-1$ matrices that have decreasing row and column sums. However, the computational problem of finding the right rearrangement of rows and columns so that the rearranged matrix has the minimum discrepancy has not, to our knowledge, been addressed before. Also, the concept of segmented nestedness and its computational analysis is new. 


\section{CONCLUSIONS}

We have studied the concepts of nestedness and segmented nestedness, arguing that they are useful also outside the field of ecology. We defined the measures that quantify how far a dataset is from being $k$-nested and studied the properties of the corresponding optimization problem. For the case $k=1$, we proposed a simple greedy algorithm that clearly outperforms existing algorithms on generated and real data. For the case $k>1$, we showed how spectral methods can be used to obtain partitions that give good results. We evaluated the methods both on synthetic and real data. The algorithms scale to moderately sized datasets.

There are obviously many open questions. One of the most interesting ones is the selection of the correct value of $k$; here we were content to use a simple heuristic, but it is interesting to study the behavior of more disciplined methods. The usefulness of the concept of segmented nestedness on real data seems clear, and additional experiments on, e.g., ecological and document data are of interest.

\section{REFERENCES}

[1] Atkins, J. E., Boman, E. G., And Hendrickson, B. A spectral algorithm for seriation and the consecutive ones problem. SIAM J. Comput. 28, 1 (1998), 297-310.

[2] Atmar, W., and Patterson, B. Nestedness temperature calculator.

[3] Atmar, W., And Patterson, B. On the measure of order and disorder in ecological communities on archipelagos. Oecologia 96 (1993), 539-547.

[4] Bascompte, J., Jordano, P., Melian, C., And Olesen, J. The nested assembly of plant-animal mutualistic networks. Proceedings of the National Academy of Sciences 100 (2003), 9383-9387.

[5] Brualdi, R., and Sanderson, J. Nested species subsets, gaps, and discrepancy. Oecologia 119, 2 (1999), 256-264.

[6] Brualdi, R. A., And Shen, J. Discrepancy of Matrices of Zeros and Ones. Electr. J. Comb. 6 (1999).

[7] Cutler, A. The dynamics of nested patterns of species distribution. Biodiversity dynamics.

[8] Cutler, A. Nested biotas and biological conservation: metrics, mechanisms, and meaning of nestedness. Conserv. Biol. 5 (1991), 496-505.

[9] Cutler, A. Nested biotas and biological conservation: metrics, mechanisms, and meaning of nestedness. Landscape and Urban Planning 28 (1994), 73-82.

[10] Ding, C. H. Q., AND He, X. Linearized cluster assignment via spectral ordering. In ICML (2004).
[11] Donlan, C., Knowlton, J., Doak, D., And Biavaschi, N. Nested communities, invasive species and holocene extinctions: evaluating the power of a potential conservation tool. Oecologia 145, 3 (2005), 475-485.

[12] Duda, R., Hart, P., And Stork, D. Pattern Recognition. Wiley, 2001.

[13] Gionis, A., Mannila, H., Mielikäinen, T., And Tsaparas, P. Assessing data mining results via swap randomization. In $K D D$ (2006), pp. 167-176.

[14] Ibanez, J., Caniego, J., And Garcia-Alvarez, A. Nested subset analysis and taxa-range size distributions of pedological assemblages: implications for biodiversity studies. Ecological Modelling 182, 3/4 (2005), 239-256.

[15] Kleinberg, J., Papadimitriou, C., And Raghavan, P. Segmentation problems. Journal of the ACM (2004), 263-280.

[16] Koren, Y., And Harel, D. Multi-scale algorithm for the linear arrangement problem, 2002.

[17] Natanzon, A., Shamir, R., And Sharan, R. Complexity classification of some edge modification problems. Discrete Applied Mathematics 113, 1 (2001), 109-128.

[18] NG, A. Y., Jordan, M. I., And Weiss, Y. On spectral clustering: Analysis and an algorithm. In NIPS (2001), pp. 849-856.

[19] Patterson, B., And Atmar, W. Nested subsets and the structure of insular mammalian faunas and archipelagos. Biological Journal of the Linnean Society 28, 1-2 (1986).

[20] Pothen, A., Simon, H., And Wang, L. Spectral nested dissection. Tech. Rep. CS-92-01, 1992.

[21] Rodriguez-Girones, M. A., and Santamaria, L. A new algorithm to calculate the nestedness temperature of presence-absence matrices. Journal of Biogeography 33, 5 (2006), 924-935.

[22] Spielman, D. A., And Teng, S.-H. Spectral partitioning works: Planar graphs and finite element meshes. In FOCS (1996), pp. 96-105.

[23] Wright, D., Patterson, B. D., Mikkelson, G. M., Cutler, A., And Atmar, W. A comparative analysis of nested subset patterns of species composition. Oecologia 113 (1998), 1-20.

[24] Wright, D., And Reeves, J. On the meaning and measurement of nestedness in species assemblages. Oecologia 92 (1992), 257-264.

[25] Yannakakis, M. The node-deletion problem for hereditary properties is NP-complete. J. Comput. System Sci. 20 (1980), 219-230.

[26] Yannakakis, M. Computing the Minimum Fill-In is NP-Complete. SIAM Journal on Algebraic and Discrete Methods 2, 1 (1981), 77-79. 